cleaved down to the mRNA 3 ' terminus. However, no evidence for such extended $3^{\prime}$ termini in the normal gene has been obtained, unlike poly(A)-containing mRNAs. The histone genes may have a similar termination mechanism to other pol II genes, but simply miss out the additional endonucleolytic and polyadenylation steps - presumably due to the absence of a suitably positioned AAUAAA sequence. Such a theory seems more plausible given that the same RNA polymerase enzyme transcribes both types of gene. This model will soon be put to the test when the true termination sites of several different pol II genes (that encode poly(A) containing mRNAs) are mapped and sequenced. These genes may possess a sequence identical or similar to the histone termination signal. Unfortunately, most gene sequence studies extend only a few hundred base pairs beyond the poly(A) site. If termination occurs over $1 \mathrm{~kb} 3^{\prime}$ to the poly(A) site, as appears to be the case with mouse $\beta$-globin, then possible termination signals will not yet have been sequenced.

Yeast pol II genes provide yet another variation. Recent studies on a naturally occurring mutant of the yeast Iso-1cytochrome $c$ gene reveal transcripts that are much longer than in wild-type yeast, due to extended $3^{\prime}$ termini ${ }^{13}$. Detailed structural analysis reveals that the mutant gene contains a 38 base pair deletion at the $3^{\prime}$ end of the mRNA sequence. Sequence comparison of all characterized yeast pol II genes shows that the deleted region includes a sequence homology present in all the yeast genes but different from AAUAAA. Curiously, in this mutant all the 3'-extended RNA transcripts are polyadenylated as is the wild-type mRNA. This lack of specificity as to which $\mathbf{3}^{\prime}$ end is adenylated is clearly different from the situation in higher eukaryotes. Presumably termination and polyadenylation are coupled events, so that only pol II transcripts end with poly(A) tails. It would appear that yeast pol II genes are intermediate between higher eukaryotic histone genes and other pol II genes encoding poly(A)-containing mRNAs.

1. McKnight, S.L., Gavis, E.R., Kingsbury, R. \& Axel, R. Cell 25, 385 (1981).

2. Mellon, P., Parker, U., Gluzman, Y. \& Maniatis, T Cell 27, 279 (1981)

3. Grosveld, G.C., de Boer, E., Shewmaker, C.K. \& Flavell, R.A. Nature 295, 120 (1981)

4. Proudfoot, N.J. \& Brownlee, G.G. Nature 263, 211 (1976)

5. Fitzgerald, M. \& Shenk, T. Cell 24, 251 (1981).

6. Flint, S.J. \& Broker, T.R. in DNA Tumour Viruses Vol. 9 (ed. Tooze, J.) 433 (Cold Spring Harbor Laboratory, New York, 1981).

7. Setzer, D.R., McGrogan, M., Nunberg, J.H. \& Schimke, R.T. Cell 22, 361 (1980).

8. Early, P. Cell 20, $313(1980)$

9. Rogers, J. et al. Cell 20, 303 (1980).

10. Hofer, E. \& Darnell, J.E. Jr Cell 23, 585 (1981)

I. DNA Tumour Viruses (ed. Tooze, J.) (Cold Spring Harbor aboratory, New York, 1981).

12. Birchmeier, C., Grosschedl, R. \& Birnstiel, M. Cell 28, 739-745 (1982).

13. Zaret, K.S. \& Sherman, F. Cell 28, 563 (1982)

\title{
Two different viral transforming proteins bind the same host tumour antigen
}

\section{from David Lane and Ed Harlow}

A RECENT publication ${ }^{1}$ by Sarnow and his colleagues shows that in adenovirustransformed mouse cells, a host protein, p53, becomes tightly associated with a 58,000 -molecular weight product of the adenovirus $\mathrm{E} 1 \mathrm{~b}$ region - one of the regions whose product is responsible for the transforming capability of adenovirus. What makes the finding of particular interest is that in SV40-transformed mouse cells, p53 also binds to the SV40-coded large $\mathrm{T}$ antigen ${ }^{2,3}$, expression of which is thought sufficient to transform rodent cells. That proteins from unrelated DNA tumour viruses should both form a stable highly specific complex with p53 (ref. 4) immediately suggests the possibility of a common function in transformed cells for either the viral proteins or the complexes themselves.

Interest in p53 has not come solely from

David Lane is in the Eukaryotic Molecular Genetics Group, Imperial College of Science and Technology, London SW7 $2 A Z$ and Ed Harlow is in the Molecular Virology Laboratory, Imperial Cancer Research Fund Laboratories, London WC2A $3 P X$. tumour virology, but also from the discovery of antibodies to p53 in sera from animals with a wide variety of tumours. Anti-p53 antibodies were first detected almost simultaneously in two separate fields. DeLeo and co-workers reported that a proportion of sera from mice with methylcholanthrene-induced tumours had anti-p53 antibodies 5 while workers with SV40 reported that $p 53$ could be immunoprecipitated with sera from animals with tumours of SV40-transformed cells ${ }^{2,3}$. Subsequently, anti-p53 antibodies were found in mice with Abelson-induced leukaemia ${ }^{6}$. Why this host protein should become immunogenic in these animals is not clear but the presence of anti-p53 antibodies in tumour sera is not restricted to experimental animals. About 10 per cent of sera from human breast cancer patients also contain anti-p53 activity, while no activity against p53 can be detected in sera from normal patients ${ }^{7}$.

It is commonly said that the level of p53 is higher in transformed cells than in normal cells $5,8,9$. Levels of p53 clearly increase following SV40 transformation, probably because of stabilization by binding to large $T$ antigen: in mouse 3T3 cells, p53 has a half life of approximately 20 min which increases to more than $24 \mathrm{~h}$ in SV40-transformed $3 \mathrm{~T} 3$ cells ${ }^{10}$.

A similar set of observations might now be expected in adenovirus-transformed cells. Reports of metabolic labelling and immunoprecipitation experiments on a wide variety of non-SV40-transformed cells have suggested that they have higher levels of p53 than their non-transformed counterparts. Increased levels of p53 labelling have also been reported to follow lectin stimulation of lymphocytes ${ }^{11}$ and to be associated with explants of early embryos ${ }^{12}$.

These reports are consistent with the hypothesis that increased levels of p53 accompany transformation or rapid cell growth but it must be remembered that the incorporation of radiolabelled precursors into immunoprecipitated p53 may not be an accurate measure of the total protein levels of p53. For example, as judged by radiolabelled p53, SV40-transformed mouse cells have two to three times more p53 than mouse F9 teratocarcinoma cells, but when tested in a radioimmunoassay for total protein, F9 cells have approximately 50 -fold less p53 than the SV40transformed cells ${ }^{13}$. Until an accurate assay for total cold p53 is widely used, the claim that elevated levels of p53 frequently accompany transformation will have to be held in some doubt.

Although the ability to label p53 may be reduced in normal cells, careful examination has shown that p53 can be found in almost all cell cultures. The only known exceptions to this rule are the human tumour cell lines $\mathrm{HeLa}$ and $\mathrm{EJ}^{9}$. No detectable levels of $\mathrm{p} 53$ have been found in these cells, and if these negative data prove correct, p53 cannot be essential for cell viability. Likewise, p53 does not represent a universal marker for transformation as it is present in normal cells and absent from some transformed cells.

Although there are very few clues to understanding the role of $\mathrm{p} 53$ in normal or transformed cells, knowing what viral processes are dependent or altered on binding to p53 is probably one of the best avenues to determining its function. The fact that two DNA tumour viruses both produce proteins that complex p 53 should greatly aid this search and may provide a clue to the immunogenicity of p53 in animals with cancer.

\footnotetext{
1. Sarnow, P. et al. Cell 28, 38 (1982)

2. Lane, D. \& Crawford, L. Nature 278, 261 (1979).

3. Linzer, D. \& Levine, A. Cell 17, 43 (1979).

4. McCormick, F. \& Harlow, E. J. Virol. 34, 213 (1980)

. Deleo, A. et al. Proc. natn. Acad. Sci. U.S.A. 76, 2420 (1979)

6. Rotter, V. et al. J. Virol. 36, 547 (1980)

7. Crawford, L., Pim, D. \& Bulbrook (in preparation)

8 Rotter, V., Boss, et al. J. Virol. 38, 336 (1981)

9. Crawford. L. et al. Proc. natn. Acad. Sci. U.S.A. 78, 41 (1981)

10. Oren, M. et al. Molec. cell. Biol. 1, 101 (1981)

11. Milner, J. \& Milner, S. Virology 112, 785 (1981).

2. Chandrasekaran, K. et al. Proc. natn. Acad. Sci. U.S.A. 78,6953 (1981).

13. Benchinol, S. (in preparation).
} 